\title{
Os efeitos dos gastos públicos em educação, saúde e trabalho no desempenho do índice FIRJAN de Desenvolvimento Municipal em Cidades do Estado de São Paulo
}

The effects of public spending on education, health and work on the performance of the FIRJAN Municipal Development Index in Cities in the State of São Paulo

Efectos del gasto público en educación, salud y trabajo sobre el desempeño del índice de desarrollo municipal FIRJAN en las Ciudades del Estado de São Paulo

Recebido: 28/12/2021 | Revisado: 03/01/2022 | Aceito: 10/01/2022| Publicado: 12/01/2022

\author{
Janayna Arruda Barroso \\ ORCID: https://orcid.org/0000-0001-6838-5937 \\ Universidade Federal do Piauí, Brasil \\ Universidade Municipal de São Caetano do Sul, Brasil \\ E-mail: janaynaarruda@ymail.com \\ Alexandre Wállace Ramos Pereira \\ ORCID: https://orcid.org/0000-0003-0396-7957 \\ Universidade Federal de Campina Grande \\ Universidade Municipal de São Caetano do Sul, Brasil \\ E-mail: alexandre.ufcg.adm@gmail.com \\ Renato Emanuel Gomes da Silva \\ ORCID: https://orcid.org/0000-0001-6438-7142 \\ Universidade Municipal de São Caetano do Sul, Brasil \\ E-mail: renatoemmanuel@hotmail.com \\ Luís Paulo Bresciani \\ ORCID: https://orcid.org/0000-0002-6028-4493 \\ Fundação Getúlio Vargas, Brasil \\ Universidade Municipal de São Caetano do Sul, Brasil \\ E-mail: luis.bresciani@online.uscs.edu.br \\ Leandro Campi Prearo \\ ORCID: https://orcid.org/0000-0002-6039-1280 \\ Universidade Municipal de São Caetano do Sul, Brasil \\ E-mail: leandro.prearo@online.uscs.edu.br
}

\begin{abstract}
Resumo
Este artigo avalia as correlações existentes ou inexistentes entre os gastos públicos das funções de governo (Educação, Saúde e Trabalho) de cidades paulistas e os resultados do Índice FIRJAN de Desenvolvimento Municipal (IFDM), utilizando a técnica estatística de análise descriminante, a fim de verificar a influência das variáveis numéricas (despesas públicas) sobre uma variável categorizada (IFDM Geral), envolvendo testes estatísticos, por meio do software SPSS (Statistical Package for the Social Sciences). Essencialmente, consistiu em coletar informações no banco de dados das Finanças dos Municípios do Brasil (FINBRA) a respeito dos gastos públicos realizados nos períodos de 2013 a 2016(últimos dados divulgados no FINBRA) em607 municípios pesquisados nas três funções de governo mencionadas. Adicionalmente, foram coletadas informações sobre a população de cada localidade junto ao Instituto Brasileiro de Geografia e Estatística (IBGE), bem como os resultados do IFDM de cada município, também nos anos especificados acima, na base de dados da Federação das Indústrias do Rio de Janeiro (FIRJAN). Os resultados apontaram a inexistência de correlação entre os gastos das funções de governo Educação e Saúde e o IFDM, sendo somente a variável Despesa - Educação significante para o modelo, ou seja, somente ela possui impacto na classificação. Sugere-se para pesquisas futuras que seja realizado uma análise discriminante do IFDM, separando os índices por suas dimensões, relacionando-se com as funções de governo em separado.
\end{abstract}

Palavras-chave: Gastos públicos; Municípios paulistas; Desenvolvimento municipal; Índice FIRJAN.

\begin{abstract}
This article evaluates the existing or non-existent correlations between public expenditures of government functions (Education, Health and Work) in São Paulo cities and the results of the FIRJAN Municipal Development Index (IFDM), using the statistical technique of discriminant analysis, in order to verify the influence of numerical variables (public expenditures) on a categorized variable (General IFDM), involving statistical tests, using the SPSS (Statistical Package for the Social Sciences) software. Essentially, it consisted of collecting information in the Brazilian Municipal Finance Database (FINBRA) regarding public expenditures carried out in the periods 2013 to 2016 (last data released in FINBRA) in 607 municipalities surveyed in the three government functions mentioned. Additionally,
\end{abstract}


information on the population of each location was collected from the Brazilian Institute of Geography and Statistics (IBGE), as well as the results of the IFDM for each municipality, also in the years specified above, in the database of the Federation of Industries of Rio de January (FIRJAN). The results pointed to the inexistence of correlation between the expenses of the Education and Health government functions and the IFDM, with only the variable Expenditure Education being significant for the model, that is, only it has an impact on the classification. It is suggested for future research that a discriminant analysis of the IFDM be carried out, separating the indices by their dimensions, relating to the government functions separately.

Keywords: Public spending; São Paulo municipalities; Municipal development; FIRJAN Index.

\section{Resumen}

Este artículo evalúa las correlaciones existentes o inexistentes entre el gasto público de las funciones gubernamentales (Educación, Salud y Trabajo) en las ciudades de São Paulo y los resultados del Índice de Desarrollo Municipal FIRJAN (IFDM), utilizando la técnica estadística de análisis discriminante, con el fin de verificar la influencia de variables numéricas (gasto público) sobre una variable categorizada (IFDM General), involucrando pruebas estadísticas, utilizando el software SPSS (Statistical Package for the Social Sciences). Básicamente, consistió en recopilar información en la Base de Datos de Finanzas Municipales de Brasil (FINBRA) sobre el gasto público realizado en los períodos 2013 a 2016 (último dato publicado en FINBRA) en 607 municipios encuestados en las tres funciones gubernamentales mencionadas. Adicionalmente, se recopiló información sobre la población de cada localidad del Instituto Brasileño de Geografía y Estadística (IBGE), así como los resultados del IFDM para cada municipio, también en los años especificados anteriormente, en la base de datos de la Federación de Industrias. de Rio de enero (FIRJAN). Los resultados apuntaron a la inexistencia de correlación entre los gastos de las funciones de gobierno de Educación y Salud y el IFDM, siendo solo la variable Gasto - Educación significativa para el modelo, es decir, solo tiene impacto en la clasificación. Se sugiere para futuras investigaciones que se realice un análisis discriminante del IFDM, separando los índices por sus dimensiones, relacionando las funciones de gobierno por separado.

Palabras clave: Gasto público; Municipios de São Paulo; Desarrollo municipal; Índice FIRJAN.

\section{Introdução}

A partir da promulgação da Constituição Federal de 1988, o Brasil presenciou um intenso processo de descentralização política, administrativa e fiscal, buscando conferir aos municípios uma maior autonomia para a formulação e implementação de políticas públicas no âmbito local, bem como para decidir sobre a melhor forma de alocação e gestão dos gastos públicos referentes a essas políticas (Arretche, 1999; Ipea, 2012; Da Rosa et al. 2021; Soares, Raupp \& Tezza, 2021).

Esse processo tornou evidente o papel exercido pelo estado brasileiro, o que reforçou tanto a necessidade de promoção do desenvolvimento nacional, quanto a melhoria do atendimento às demandas da população por meio da garantia ao acesso aos serviços públicos e, mais especificadamente em se tratando dos entes subnacionais, exigindo mais efetividade no planejamento das gestões municipais (Monteiro Neto et al, 2017; Forte \& Vieira, 2021).

No entanto, a descentralização também levou a muitas questões sobre a realidade das administrações locais. Uma dessas questões se refere à alocação dos gastos públicos (Magalhães, 1997) e das grandes assimetrias regionais, as quais exigem ações tem educação, saúde entre outras ações (Höfling et al., 2001).

A esse respeito, algumas pesquisas se propuseram a estudar a questão dos gastos públicos e sua relação com o nível de desenvolvimento humano (Slomski \& Scarpin, 2006; Oliveira, 2016), bem como com outros indicadores sociais (Santos Filho, 2010; Avelino et al., 2013 Soares et. al., 2020; Dantas \& Silva, 2019; Jesus et al., 2019; Castro et al., 2021; Lepchak et al., 2021) e ainda a sua relação com índices de desenvolvimento e crescimento econômico (Silva \& Azevedo, 2018)

Em se tratando desses índices, é possível constatar a existência de diversos deles, tais como o Índice de Desenvolvimento Humano Municipal (IDHM), o Índice de Desenvolvimento da Educação Básica (IDEB) e o Índice da Federação das Indústrias do Estado do Rio de Janeiro (FIRJAN) de Desenvolvimento Municipal (IFDM), entre outros.Neste sentido, o IFDM merece destaque, por ser um estudo anual realizado em todo o Brasil, desde o ano de 2008, o qual avalia o desenvolvimento humano, econômico e social municipal, tendo importante contribuição no auxílio do controle social da gestão, por parte da população, como também por parte dos gestores públicos, os quais podem compreender melhor as áreas 
que os investimentos estão gerando retorno à sociedade, bem como aquelas que necessitam de mais recursos para garantir o atendimento às demandas da população (Firjan, 2020).

Estudos anteriores buscaram estudar o Indice FIRJAN de Desenvolvimento Municipal e sua comparação com a evolução do Produto Interno Bruto (PIB) Municipal (Silva \& Brito, 2019), por exemplo. Ainda, diversos outros podem ser citados, apresentando diferentes escopos de abrangência territorial e uma diversidade de objetivos.

Dentre os vários objetivos propostos, alguns merecem destaque, a saber: avaliar a efetividade ou inércia do desenvolvimento de municípios do Estado do Rio Grande do Norte a partir do Indice FIRJAN (Silva \& Azevedo, 2018; Silva, Oliveira, \& Carniello, 2021); estudar a relação dos recursos do Programa Bolsa Família (PBF) com o Índice FIRJAN dos municípios que compõem os Conselhos Regionais de Desenvolvimento (COREDEs) do Estado do Rio Grande do Sul; identificar a relação entre algumas variáveis contábeis selecionadas, condicionantes do Índice FIRJAN em municípios brasileiros de grande porte (De Andrade et al., 2019); identificar as variáveis contábeis condicionantes do IFDM das capitais brasileiras (Avelino et al., 2013); analisar a relação entre o crescimento e o desenvolvimento econômico dos municípios do Estado do Pará, utilizando as bases de dados do Índice FIRJAN e, por fim, analisar a influência dos gastos Públicos no crescimento e desenvolvimento econômico nos municípios de Santa Catarina (De Sousa et al., 2020).

No que se refere ao Estado de São Paulo, foi identificado um estudo realizado no ano de 2019, com uma descrição crítica dos principais índices que se propõem a medir o desenvolvimento dos municípios do Estado de São Paulo, apontando suas virtudes, imperfeições e limitações, quais sejam: o Índice de Desenvolvimento Humano Municipal (IDHM), o Índice Firjan de Desenvolvimento Municipal (IFDM) e o Índice Paulista de Responsabilidade Social (IPRS). O trabalho, no entanto, faz uma descrição crítica dos três principais índices de forma descritiva, efetuada apenas por meio de pesquisa bibliográfica (Bellingieri, 2019).

Considerando esses estudos citados, embora a maioria tenha associado o IFDM com vários indicadores, não há em nenhum deles a análise da relação entre o desempenho do Índice FIRJAN de Desenvolvimento Municipal com os gastos específicos em três funções de governo específicas (Educação, Saúde e Trabalho), em âmbito municipal. Além disso, observase que a maior parte das pesquisas se concentrou em amostras menores, abarcando capitais ou uma região específica do país. Sendo assim, uma análise envolvendo todos os municípios do Estado de São Paulo pode se configurar como uma boa oportunidade para contribuir com evidências sobre a dinâmica dos gastos públicos em funções de governo de maior impacto na vida da população, como é o caso das áreas de educação, saúde e emprego e renda.

Sendo assim, a análise da relação entre gastos públicos e os seus efeitos no desempenho do IFDM, como propõe esse trabalho, pode ser relevante aos gestores públicos locais no sentido de permitir avaliações e tomadas de decisões, bem como norteá-los para obter um melhor desenvolvimento municipal, em especial para a concretização das políticas relativas às funções governamentais de educação, saúde e trabalho. Ademais, a pesquisa se justifica ao contribuir com outra abordagem territorial, especialmente ao englobar 607 municípios do Estado de São Paulo, buscando avaliar os reflexos dos gastos governamentais por função da despesa pública no referido índice FIRJAN de desenvolvimento municipal.

Esta pesquisa procura responder a seguinte questão: Qual a relação entre os gastos em educação, saúde e trabalho dos municípios do Estado de São Paulo e o desempenho verificado no IFDM dessas cidades?

Guiado por essa pergunta, este estudo tem como objetivo avaliar as correlações existentes ou inexistentes entre os gastos públicos das funções de governo selecionadas (Educação, Saúde e Trabalho) das cidades paulistas e os resultados do IFDM. Portanto, quanto ao aspecto territorial, a pesquisa se limita às cidades localizadas no Estado de São Paulo. Quanto ao conteúdo, ficará delimitada aos gastos com as políticas municipais de educação, saúde e trabalho e ao IFDM geral analisados por função per capta. Quanto ao aspecto temporal, analisou-se as informações referentes aos anos de 2013, 2014, 2015 e 2016 , 
sendo estes ou últimos dados divulgados no site Finanças dos Municípios do Brasil FINBRA, conferindo assim, maior dinâmica da evolução dos gastos públicos e seus efeitos nos resultados do IFDM.

A partir dessas considerações, apresenta-se as seguintes hipóteses: (H0) Existe uma relação positiva entre a evolução dos gastos com educação, saúde e trabalho com o desempenho do IFDM e (H1) Não existe uma relação positiva entre os gastos com educação, saúde e trabalho com o desempenho do IFDM dos municípios paulistas.

\section{Aporte Teórico}

Para esta fundamentação teórica, buscou-se abordar conceitos sobre gastos públicos no sentido de contemplar as funções de governo: saúde, educação e trabalho, como também, conceitos do índice Firjan de Desenvolvimento Municipal (IFDM).

\subsection{Gastos públicos, desenvolvimento municipal e indicadores sociais}

A implementação de políticas públicas, a eficiência dos gastos públicos, bem como os resultados das ações do governo vem ganhando destaque nos últimos anos, atraindo a atenção de pesquisadores, gestores, formuladores de políticas públicas e dos mais diversos profissionais em todo o mundo. Neste sentido, há um consenso de que os gastos públicos interferem diretamente na vida dos cidadãos e estes, por conseguinte, podem promover a melhoria da dignidade da pessoa humana e o atendimento às demandas da sociedade (Matias-Pereira, 2012; Da Silva Gomes, 2019; Sun, Andrews, 2019).

No Brasil, a forma e a decisão de gastar, estão sujeitas a uma série de fontes normativas, as quais descrevem prescrições para o gestor público, sendo a principal delas, a Constituição Federal de 1988. Desse modo, a decisão de gastar vincula-se mais diretamente ao Plano Plurianual (PPA), à Lei Orçamentária Anual (LOA) e à Lei de Diretrizes Orçamentárias (LDO), as quais estão previstos os objetivos e metas, as fontes de receita, as dotações e às fases de execução das despesas públicas. Ainda, a determinação por gastos de governo vincula-se às normas gerais de direito financeiro, especialmente o constante na Lei $\mathrm{n}^{\circ}$ 4.320/64, bem como aos princípios constitucionais da despesa pública (Brasil, 1964; Brasil, 1988, Procopiuck, Machado, Rezende \& Bessa, 2007).

Neste sentido, um fato importante a se abordar é o de que os gastos públicos possuem função social, tendo a finalidade de fazer valer os direitos sociais garantidos na Constituição da República. Assim, cabe ao Estado a obrigação de implementar esforços para que os gastos públicos sejam manejados no sentido de fazer cumprir os direitos sociais garantidos pela Carta Magna (Comparato, 2001; Massaú \& Rafagnin, 2021).

De um modo geral, no setor público, os termos "gasto" e "despesa" são utilizados indistintamente para designar o mesmo objeto. Segundo Torres (1996), despesa pública pode ser definida como a soma dos gastos realizados pelo Estado para a realização de obras e para a prestação de serviços públicos. Gasto público, de acordo com Rezende Cunha (2010), é toda despesa executada pelo município com recursos de sua receita. Riani (2000), Giacomoni (2003) e Rezende (2006), enfatizam que os gastos públicos são as despesas governamentais necessárias para o provimento (desenvolvimento e manutenção) de bens e serviços a serem disponibilizados à sociedade. Segundo Lapatza (2004), uma definição sintética de gasto público é todo aquele realizado por um ente público. Para isso, é necessário que o mesmo esteja autorizado no orçamento do ente que o realiza. Ademais, qualquer gasto deve estar devidamente relacionado ao objeto, bem como a definição dos limites e a norma jurídica que o disciplina.

No Brasil, não há uma definição legal de despesa pública, pois embora a Lei n 4.320/64 apresente em seus artigos 12 e 13 a classificação das despesas, não traz sua definição específica. A classificação citada pela referida lei ajuda na compreensão do que seja despesa orçamentária. 
A partir das conceituações apresentadas, é possível enfatizar que o gasto público tem vinculação com o atendimento às necessidades coletivas. Isto posto, compreende-se este como gastos mais diretamente voltados ao atendimento das demandas sociais da população, ou seja, à melhoria das condições de vida do cidadão e são considerados geralmente como gastos sociais. Estes incluem ações governamentais ligadas à saúde, educação, seguridade e assistência social, emprego, saneamento, entre outros (Rezende Cunha, 2010; Freitas, 2019; Goulart et al., 2020; Pinheiro et al., 2020).

Considerando as ações governamentais desenvolvidas pelo poder público, por meio das políticas públicas, para atender às demandas sociais da população, a Constituição Federal de 1988, em seu art. 197 descreve os direitos sociais à saúde e à educação (Do Brasil, 1988). Essas funções, portanto, integram o núcleo essencial dos direitos fundamentais de todos os brasileiros, para as quais o texto constitucional expressa percentuais mínimos da receita arrecadada pelos entes governamentais para que seja investido em políticas de educação e saúde.

A saúde se insere na órbita dos direitos sociais constitucionalmente garantidos, assim como a educação. O texto constitucional assegura:

Art.196. A saúde é direito de todos e dever do Estado, garantido mediante políticas sociais e econômicas que visem à redução do risco de doença e de outros agravos e ao acesso universal e igualitário às ações e serviços para sua promoção, proteção e recuperação.

A respeito da função educação, a Constituição de 1988 descreve:

Art. 205. A educação, direito de todos e dever do Estado e da família, será promovida e incentivada com a colaboração da sociedade, visando ao pleno desenvolvimento da pessoa, seu preparo para o exercício da cidadania e sua qualificação para o trabalho (BRASIL, 1988).

Alguns autores enfatizam que elevados níveis de educação tendem a melhorar o nível de capital humano de um país ao gerar crescimento econômico no longo prazo (Whiteley, 2000). Sendo assim, considera-se que os gastos com educação são os que mais geram crescimento, ou melhor, é o investimento público com educação que eleva o nível de capital humano que, consequentemente, promove o crescimento da economia (Zoghbi et al., 2009).

Embora seja consenso que a adoção de práticas que levem a uma melhor gestão dos gastos com educação, buscando ampliar e melhorar as saídas de serviço educacional público à população (Diel et al., 2014), estudos revelam que não existe uma relação entre os gastos com educação e os índices de eficiência (Faria et al., 2008; Will, 2014; Dias et al., 2014)

Considerando o exposto, os índices constitucionais de aplicação obrigatórios pelos municípios são os seguintes, de acordo com a Constituição Federal de 1988:

- Aplicação de no mínimo $15 \%$ das receitas de impostos e transferências em ações e serviços públicos de Saúde (índice Saúde 15\%);

- Aplicação de no mínimo $25 \%$ das receitas de impostos e transferências em educação e desenvolvimento do ensino (Índice Educação 25\%);

- Aplicação de no mínimo $60 \%$ dos recursos do Fundo de Manutenção e Desenvolvimento da Educação Básica (FUNDEB) em valorização e remuneração dos profissionais do magistério (Índice FUNDEB 60\%).

Embora o trabalho seja uma função de impacto para a melhoria das condições de vida da população, inclusive considerado como direito social, a Constituição não especifica percentuais para gastos com políticas referentes ao emprego e renda, ficando essa atividade como um fator de discricionariedade por parte de cada ente governamental.

Diversos estudos, nos últimos anos, analisaram a relação entre gastos do governo e o desenvolvimento de municípios. Entre os estudos destacam-se o trabalho de Rezende et al., (2005), cujo objetivo foi analisar as variáveis condicionantes entre 
as políticas públicas e o nível de desenvolvimento humano dos municípios do Estado de São Paulo; o trabalho de Scarpin e Slomski (2007) buscando analisar os fatores contábeis condicionantes do Índice de Desenvolvimento Humano Municipal (IDH-M) na dimensão renda em municípios paranaenses.

Também merecem destaque os estudos de Santos Filho (2010), observando a relação entre indicadores sociais e a alocação de recursos públicos; Oliveira (2016) investigou se as despesas públicas municipais são eficientes quanto a proporcionar desenvolvimento humano dos municípios brasileiros; Avelino et al. (2013) discutiram a administração das contas públicas municipais e as respectivas informações contábeis e o resultado no nível de desenvolvimento municipal por meio do estudo de indicadores; Silva e Azevedo (2018) buscaram avaliar efetividade ou inércia no desenvolvimento dos municípios do Rio Grande do Norte; Silva et al. (2019) analisaram a relação entre crescimento e desenvolvimento econômico no município de São José dos Campos; e por fim, Sales (2019) discute a evolução do desenvolvimento social e econômico em municípios receptores de parques eólicos no Brasil

É possível constatar, a partir da análise dos trabalhos acima, que os gastos do governo em diferentes funções da despesa podem se refletir na melhoria dos indicadores sociais, os quais, por sua vez, são capazes de promover impactos na sociedade, uma vez que possuem a capacidade de informar os gestores públicos a respeito dos resultados de suas estratégias de alocação de recursos nos indicadores). sociais e, ao mesmo tempo, permitir que a população avalie o trabalho daqueles que foram escolhidos para gerir os recursos públicos (De Andrade et al., 2019

Em razão da importância dos gastos públicos no desenvolvimento social e econômico, segundo Rezende, Slomski e Corrar (2005), tornou-se necessário o desenvolvimento de métricas que possibilitassem a mensuração dos investimentos sociais e seus impactos na sociedade, bem como avaliar o desenvolvimento dos países. Desse modo, em se tratando de indicadores sociais, a criação do Índice de Desenvolvimento Humano (IDH), representou uma grande contribuição nesse sentido (Scarpin, Slomski, 2007), cuja metodologia deu base para a criação de outros indicadores, como é o caso do Índice FIRJAN de Desenvolvimento Municipal (IFDM), descrito na próxima seção.

\section{2 Índice FIRJAN de Desenvolvimento Municipal}

A promulgação da Constituição Federal de 1988 foi um marco no processo de descentralização política e fiscal no Brasil, o que levou aos municípios brasileiros a terem uma maior autonomia na condução de suas políticas públicas locais (Ipea, 2012). Portanto, a partir desse período os gestores locais começaram a ter autonomia para decidir qual a melhor forma de gerir os recursos públicos de forma a atender as necessidades da população.

Constantemente, a renda média e o Produto Interno Bruto (PIB) per capita são utilizados como indicadores para avaliar o desenvolvimento econômico e crescimento de certa região. Entretanto, os indicadores mencionados anteriormente não conseguem medir a qualidade de vida da população de forma efetiva, especialmente ao que diz respeito ao aspecto da distribuição de renda (Ipea, 2012). Não obstante, a criação de um índice que pudesse monitorar o desenvolvimento socioeconômico dos municípios foi crucial para avaliar a gestão de investimentos públicos por parte dos seus gestores.

Em 2008 foi criado, no Brasil, o Índice Firjan de Desenvolvimento Municipal (IFDM), que tem como objetivo medir o desenvolvimento socioeconômico dos municípios. O IFDM é um indicador composto que aborda com igual ponderação três áreas, a saber: saúde, educação e emprego e renda e vem acompanhando os municípios brasileiros desde o ano 2000 com periodicidade anual (Duarte \& Alves, 2019). Os indicadores são calculados embasados em estatísticas oficiais públicas, conforme apresentado no Quadro 1. 
Quadro 1: Componentes do IFDM por área de desenvolvimento.

\begin{tabular}{|c|c|c|c|}
\hline IFDM & Emprego e renda & $\begin{array}{r}\text { Saúde } \\
\end{array}$ & Educação \\
\hline Pilares & Mercado de trabalho formal & $\begin{array}{c}\text { Atenção básica, primeiro nível de } \\
\text { contado da sociedade com o } \\
\text { sistema de saúde }\end{array}$ & $\begin{array}{l}\text { Ensino fundamental, educação } \\
\text { infantil e qualidade de educação }\end{array}$ \\
\hline Indicadores & $\begin{array}{l}\text { - Geração e emprego formal; } \\
\text { - Taxa de formalização do } \\
\text { mercado de trabalho; } \\
\text { - Geração de renda formal; } \\
\text { - Massa salarial real do mercado } \\
\text { de trabalho formal; } \\
\text { - Desigualdades de renda no } \\
\text { trabalho formal; }\end{array}$ & $\begin{array}{l}\text { - Proporção de atendimento } \\
\text { adequado de pré-natal; } \\
\text { - Óbitos por causa mal } \\
\text { definidas; } \\
\text { - Óbitos infantis por causas } \\
\text { evitáveis; } \\
\text { - Internação sensível à atenção } \\
\text { básica. }\end{array}$ & $\begin{array}{l}\text { - Matrícula na educação } \\
\text { infantil; } \\
\text { - Abandono no ensino } \\
\text { fundamental; } \\
\text { - Distorção idade-série no } \\
\text { ensino fundamental; } \\
\text { - Docentes com ensino superior } \\
\text { no ensino fundamental; } \\
\text { - Média de horas aula diária no } \\
\text { ensino fundamental; } \\
\text { - Resultado do IDEB no ensino } \\
\text { fundamental. }\end{array}$ \\
\hline Fonte & Ministério do Trabalho (MT) & $\begin{array}{l}\text { Ministério da Saúde } \\
\text { (MS) }\end{array}$ & Ministério da Educação (MEC) \\
\hline
\end{tabular}

Legenda: Índice de Desenvolvimento da Educação Básica (IDEB). Fonte: Firjan (2018).

A partir dos indicadores acima expostos pode-se perceber que a interpretação dos resultados é simples, uma vez que a variação do índice é de 0 a 1, sabendo que quanto mais próximo de 1, maior o desenvolvimento da localidade. Os municípios são avaliados em quatro níveis de desenvolvimento, a saber: IFDM baixo, entre 0,0 e 0,4; IFDM regular, entre 0,4 e 0,6; IFDM moderado, entre 0,6 e 0,8; e alto IFDM, entre 0,8 e 1,0. Lembrando que a avaliação é apresentada em três dimensões do desenvolvimento, que são: IFDM-Saúde (IFDM-SA), Educação (IFDM-ED) e IFDM-Emprego e Renda (IFDM-ER) (FIRJAN, 2018). Vale ressaltar que também é apresentado um índice geral, o qual é mensurado a partir da média aritmética das referidas três dimensões.

O índice Firjan pode ser comparado ano a ano em um dado período de tempo, possibilitando verificar se houve de fato um progresso no desenvolvimento de um determinado município ou região, pode-se ainda, contrastar a avaliação do índice com as políticas públicas específicas direcionadas para as três dimensões avaliadas pelo índice, com o intuito de identificar se a variação positiva ou negativa do índice é por causa das políticas públicas adotadas.

Os Ministérios da Saúde, do Trabalho e da Educação divulgam seus resultados em diferentes períodos. Sendo assim, o IFDM é lançado, em geral, com defasagem temporal de dois anos em média. A edição de 2018 do IFDM, em razão de atrasos na divulgação dos dados do DataSus (braço estatístico do Ministério da Saúde) contou com os dados de 2016 para o cálculo da dimensão Saúde (Confins, 2020).

De acordo com relatório do IFDM 2016, o Brasil possui 5.570 municípios no total, no entanto, no ranking geral do IFDM de 2016 estão compreendidos 5.471 municípios brasileiros, onde vivem 99,5\% da população brasileira. Os municípios excluídos dessa foram aqueles em que os dados estavam ausentes ou insuficientes.

\section{Metodologia}

Esta pesquisa caracterizou-se como um estudo de natureza básica e, segundo seus propósitos e procedimentos técnicos, como pesquisa descritiva e documental. Quanto a forma de abordagem, classifica-se como quantitativa, valendo-se de técnicas e análises estatísticas a fim de compreender as relações entre as variáveis pesquisadas (Gil, 2018; Gray 2016; Creswell, 2021; Callado et al., 2013).

No que tange à coleta de dados, foram coletadas informações, no período de fevereiro a abril de 2021, no banco de dados das Finanças dos Municípios do Brasil (FINBRA), que contém informações sobre o volume de investimentos nas áreas de saúde, educação e trabalho, ou seja, foram levantados, os gastos públicos realizados nos período de 2013 a 2016 , pelos 
municípios pesquisados. É importante reforçar que o último exercício financeiro pesquisado foi 2016, por ser o último ano em que foram publicados os resultados do IFDM. Adicionalmente, foram coletadas informações junto ao Instituto Brasileiro de Geografia e Estatística (IBGE), como dados sobre a população. Por último, foram levantados os registros do IFDM na base de dados da Federação das Indústrias do Rio de Janeiro (FIRJAN).

A amostra pesquisada foi a não probabilística (Callado et al., 2013) e contemplou 607 municípios do total de 645 do estado de São Paulo, a intenção era pesquisar toda a população, mas não haviam dados disponíveis de todos os municípios.

Quanto às técnicas e análises estatísticas utilizadas, optou-se pela técnica estatística de análise descriminante (Levin et al., 2012), a fim de verificar a influência das variáveis numéricas (despesas públicas) sobre uma variável categorizada (IFDM Geral). Para tanto, foi necessária a realização de algumas etapas, a saber:

1. Verificação da estatística descritivas das variáveis dependentes e independentes;

2. Remoção dos outliers;

3. Verificação da normalidade das variáveis independentes por meio do teste de normalidade de Shapiro Wilk;

4. Análise discriminante múltipla.

A segunda parte, de análise quantitativa dos dados, envolveu testes estatísticos e a descrição dos resultados encontrados foi analisada por meio do software SPSS (Statistical Package for the Social Sciences).

\section{Resultados e Discussão}

Nesta seção serão apresentados todos os dados e tabelas geradas pelo SPSS, buscando verificar as hipóteses levantadas pelo presente estudo.

\subsection{Análise Discriminante}

\subsubsection{Determinação do intervalo de agrupamento do Índice FIRJAN}

Na sequência dos procedimentos, o índice FIRJAN foi segmentado em 4 intervalos e categorizado em 4 grupos conforme os quartis da distribuição, conforme ilustra a

Tabela 1.

Tabela 1: Intervalo dos valores dos percentis e determinação dos grupos.

\begin{tabular}{c|c|c}
\hline Grupo & Intervalo (percentil [\%]) & FIRJAN geral \\
\hline G1 & $0-25$ & $\leq 0,7194$ \\
G2 & $25-50$ & $0,7195-0,7637$ \\
G3 & $50-75$ & $0,7638-0,8111$ \\
G4 & $75-100$ & $\geq 0,8112$ \\
\hline
\end{tabular}

Fonte: Dados da pesquisa.

$\mathrm{Na}$ Tabela 3 está exposto o intervalo pré-determinado para aproximar o modelo. Essa categorização será utilizada de modo a facilitar a elaboração do modelo final multivariável a partir da análise discriminante.

\subsubsection{Estatística descritiva e normalidade das variáveis}

A 
Research, Society and Development, v. 11, n. 1, e47811125215, 2022

(CC BY 4.0) | ISSN 2525-3409 | DOI: http://dx.doi.org/10.33448/rsd-v11i1.25215

Tabela 2 ilustra a estatística descritiva das variáveis de entrada (métricas) referentes às despesas de cada município. 
Tabela 2: Estatística descritiva das despesas per capita.

\begin{tabular}{l|c|c|c}
\hline \multicolumn{1}{c|}{ Item } & Média & Mediana & Desvio padrão (s) \\
\hline Despesa - Saúde & 712,62 & 672,77 & 4,67 \\
Despesa - Trabalho & 2,69 & 0,00 & 0,25 \\
Despesa - Educação & 858,82 & 823,09 & 5,51 \\
\hline
\end{tabular}

Fonte: Dados da pesquisa.

Por meio dos resultados descritos na Tabela 1, percebe-se que os gastos per capita de cada município na Educação foram superiores aos demais, com uma média de valores de (R\$ 858,82), seguidos pelas despesas com Saúde (R\$ 712,62). De acordo com a Constituição Federal de 1988 são estabelecidos percentuais mínimos para gastos com Educação e Saúde. Os gastos com Educação devem ser de no mínimo $25 \%$ das receitas e impostos municipais, enquanto, os gastos com saúde devem ser de no mínimo $15 \%$, e o compromisso constitucional se reflete em alguma medida nas médias verificadas. O gasto maior em Educação previsto na Constituição pode ser um indicativo de uma relação entre o investimento em educação e o desenvolvimento econômico municipal, o que corrobora com o que foi dito por Whiteley, (2000) e Zoghbi et al. (2009) que apontam que os gastos com Educação geram maior crescimento econômico a longo prazo.

Os valores resultantes para a despesa com o Trabalho indicam valores discrepantes em função do investimento, sendo praticamente nulo o realizado no período observado (2013 a 2016). A mediana indica, de forma similar, o valor no qual se divide a distribuição no meio, ou seja, 50\% dos municípios investem acima ou abaixo do valor da mediana.

Destaca-se que os municípios em sua grande maioria no período em que foram pesquisados, não realizaram nenhum tipo de investimento na função Trabalho, o que causou a discrepância em relação aos valores em educação e saúde. Fica claro que os gestores públicos têm investido prioritariamente mais em Educação seguidos por investimento em Saúde e não têm considerado importante o investimento em Trabalho. Este comportamento pode ser justificado por não haver na Constituição Federal de 1988 nenhuma exigência de gasto na função trabalho, o que denota que os gestores públicos em sua maioria só investem no que é imposto por lei.

A normalidade das populações das variáveis realizado neste trabalho através do teste de normalidade de Shapiro-Wilk é um dado importante, conforme especificado na Tabela 2.

Tabela 3: Teste de normalidade de Shapiro-Wilk.

\begin{tabular}{l|c|c|c}
\hline & \multicolumn{3}{|c}{ Shapiro-Wilk } \\
& Estatística & gl & Sig. \\
\hline Despesa - Saude & 0,961 & 2130 & 0,000 \\
Despesa - Trabalho & 0,252 & 2130 & 0,000 \\
Despesa - Educacao & 0,973 & 2130 & 0,000 \\
\hline
\end{tabular}

Fonte: Dados da pesquisa.

A significância da análise foi inferior a 0,01, rejeitando-se a hipótese H0 e verificando-se a não-normalidade das variáveis. As variáveis estudadas apresentavam um desvio em torno da linha média (skewness) em relação à distribuição normal.

\subsubsection{Verificação da existência de correlações entre duas variáveis (bivariável)}

Para que o modelo discriminante apresente resultado positivo, é necessário que as variáveis independentes (despesas) apresentem certa correlação com a variável dependente (índice FIRJAN). Para isso, as correlações foram verificadas par-a-par e podem ser visualizadas na Figura 1. 
Figura 1: Correlação bivariável entre as despesas e o índice FIRJAN geral.

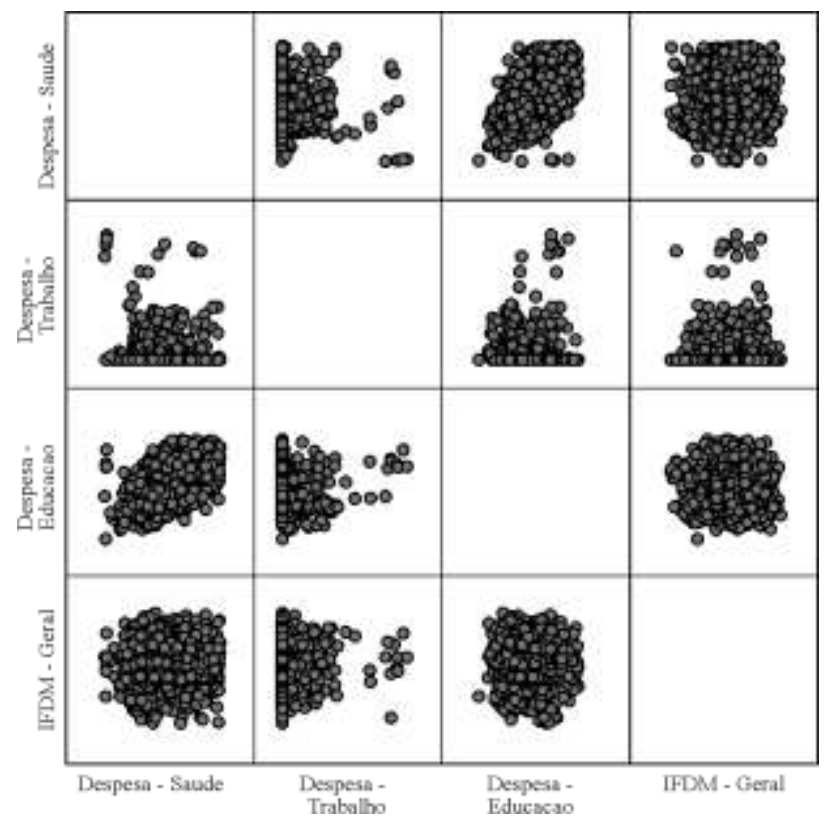

Fonte: Dados da pesquisa.

Esperava-se que a correlação entre as variáveis apresentasse uma reta diagonal, sem muita dispersão. Entretanto, observa-se uma certa dispersão e, em alguns casos, aglomeração de dados em um dos eixos. A única correlação significante é vista para as variáveis independentes, despesas com Saúde e Educação. As variáveis Saúde e Educação têm uma tendência próxima de comportamento, como inicialmente sinalizado na Tabela 1, onde foram descritas as médias e os desvios-padrão, que nas duas variáveis são bem próximos. A despesa com Trabalho apresentou correlação fraca, o que pode prejudicar no modelo final.

Com esta análise, segue-se para a verificação de multicolinearidade, que emprega a correlação de Pearson para verificar se existem correlações entre os dados. Para a análise discriminante, espera-se valores inferiores a 0,8 ou 0,9 (correlações fracas). O resultado pode ser verificado na Tabela 4.

Tabela 4: Avaliação da multicolinearidade entre as variáveis.

\begin{tabular}{|c|c|c|c|c|c|}
\hline & & Despesa - Saúde & Despesa - Trabalho & Despesa - Educação & IFDM - Geral \\
\hline \multirow[t]{3}{*}{ Despesa Saúde } & \multirow{3}{*}{$\begin{array}{l}\text { Correlação de Pearson } \\
\text { Sig. (2 extremidades) } \\
\text { N }\end{array}$} & \multirow[t]{2}{*}{1} & $-0,060^{* *}$ & $0,584^{* *}$ & $-0,015$ \\
\hline & & & 0,006 & 0,000 & 0,489 \\
\hline & & 2130 & 2130 & 2130 & 2127 \\
\hline \multirow{2}{*}{$\begin{array}{l}\text { Despesa } \\
\text { Trabalho }\end{array}$} & \multirow{2}{*}{$\begin{array}{l}\text { Correlação de Pearson } \\
\text { Sig. (2 extremidades) } \\
\text { N }\end{array}$} & $-0,060^{* * *}$ & \multirow{2}{*}{1} & $0,044^{*}$ & $0,050^{*}$ \\
\hline & & $\begin{array}{c}0,006 \\
2130\end{array}$ & & $\begin{array}{c}0,044 \\
2130\end{array}$ & $\begin{array}{c}0,022 \\
2127\end{array}$ \\
\hline \multirow[t]{2}{*}{ Despesa Educação } & \multirow{2}{*}{$\begin{array}{l}\text { Correlação de Pearson } \\
\text { Sig. (2 extremidades) } \\
\text { N }\end{array}$} & $0,584^{* *}$ & $0,044^{*}$ & 1 & $-0,049^{*}$ \\
\hline & & $\begin{array}{l}0,000 \\
2130\end{array}$ & $\begin{array}{l}0,044 \\
2130\end{array}$ & 2130 & $\begin{array}{l}0,024 \\
2127\end{array}$ \\
\hline \multirow[t]{2}{*}{ IFDM Geral } & Correlação de Pearson & $-0,015$ & $0,050^{*}$ & $-0,049^{*}$ & \multirow[t]{2}{*}{1} \\
\hline & $\begin{array}{l}\text { Sig. (2 extremidades) } \\
\mathbb{N}\end{array}$ & $\begin{array}{l}0,489 \\
2127\end{array}$ & $\begin{array}{l}0,022 \\
2127\end{array}$ & $\begin{array}{l}0,024 \\
2127\end{array}$ & \\
\hline
\end{tabular}

**. A correlação é significativa no nível 0,01 (2 extremidades).

*. A correlação é significativa no nível 0,05 (2 extremidades).

Fonte: Dados da pesquisa. 
Os números grifados na Tabela 4 indicam que não existe correlação superior aos valores de 0,8 estipulados inicialmente, o que denota que as correlações são fracas. A única correlação com destaque é observada entre as despesas de Saúde e Educação, conformeverificado anteriormente.

Baseando-se nas premissas de aplicação da técnica de análise discriminante, foram consideradas as seguintes pontos:

a) Tamanho da amostragem superior a 20;

b) Homoscedasticidade;

c) Linearidade;

d) Normalidade multivariada;

e) Ausência de multicolinariedade; e

f) Ausência de observações atípicas (outliers).

Tendo em vista que o número de amostras da análise é superior a 20 para cada condição, com 2130 amostras distribuídas em aproximadamente 607= municípios ao longo de 4 anos (2013 a 2016), optou-se por não realizar uma análise temporal (4 anos), partindo da premissa de elaborar um modelo mais assertivo com os dados de todos os anos em conjunto, ao invés de um modelo para cada ano.

O segundo critério avalia a homoscedasticidade, através de um teste de hipóteses.

Tabela 5: Teste de homoscedasticidade.

\begin{tabular}{c|c|c}
\hline \multicolumn{2}{c}{ M de Box } & 68,541 \\
\multirow{2}{*}{$\mathrm{Z}$} & Aprox. & 3,798 \\
& df1 & 18 \\
& df2 & 15926730,262 \\
\multicolumn{2}{c}{ Testa hipótese nula de matrizes de covariâncias de população igual. } \\
\hline
\end{tabular}

Fonte: Dados da pesquisa.

A hipótese nula foi rejeitada, o que significa que existem populações diferentes, indicado pela variância diferente para cada observação (heteroscedasticidade). A normalidade multivariada não foi verificada. A multicolinearidade foi verificada anteriormente na Tabela 4 e atestou-se sua ausência.

\subsubsection{Resumo das funções discriminantes canônicas}

Avaliou-se por meio do Teste Lambda de Wilks as 3 variáveis simultaneamente para verificar seus graus de diferenciação. No teste, espera-se rejeitar a hipótese nula de que as variáveis são iguais entre os grupos, obtendo Lambda menor que um e ainda, quanto menor for seu valor, mais significativa será a variável. A Tabela 6 destaca os valores da função escolhida:

Tabela 6: Resultado da estatística Lambda de Wilks.

\begin{tabular}{l|c|c|c}
\hline & Lambda de Wilks & $\mathrm{Z}$ & Sig. \\
\hline Despesa - Saúde & 0,999 & 0,731 & 0,533 \\
Despesa - Trabalho & 0,998 & 1,750 & 0,155 \\
Despesa - Educação & 0,992 & 5,396 & 0,001 \\
\hline
\end{tabular}

Fonte: Dados da pesquisa.

O teste foi significativo somente para a variável Despesa - Educação, ou seja, somente esta variável tem impacto significativo no modelo. 
À título ilustrativo, a Tabela 7 apresenta as funções de Fisher para cada grupo estudado:

Tabela 7: Coeficientes da função de classificação.

\begin{tabular}{l|c|c|c|c}
\hline & G1 & G2 & G3 & G4 \\
\hline Despesa - Saude & 0,009 & 0,009 & 0,010 & 0,010 \\
Despesa - Trabalho & 0,017 & 0,020 & 0,025 & 0,030 \\
Despesa - Educacao & 0,009 & 0,009 & 0,008 & 0,008 \\
(Constante) & $-8,430$ & $-8,937$ & $-8,386$ & $-8,301$ \\
\hline
\end{tabular}

Fonte: Dados da pesquisa.

As funções de Fisher para grupo podem ser escritas como, equação (1) a (4), respectivamente:

$$
\begin{aligned}
& \mathrm{G} 1=-8,430+0,009 \mathrm{Desp}_{\text {gande }}+0,017 \mathrm{Desp}_{\text {trab }}+0,009 \mathrm{Desp}_{\text {adu }} \\
& \mathrm{G} 2=-8,937+0,009 \text { Desp }_{\text {tzude }}+0,020 \text { Desp }_{\text {trab }}+0,009 \text { Desp } \text { edu } \\
& \text { G3 }=-8,386+0,010 \text { Desp }_{\text {gzude }}+0,025 \text { Desp }_{\text {trab }}+0,008 \text { Desp } \text { edu } \\
& \mathrm{G} 4=-8,301+0,010 \text { Desp }_{\text {taude }}+0,030 \text { Desp }_{\text {trab }}+0,00 \text { Desp } \mathrm{pdu}_{\mathrm{adu}}
\end{aligned}
$$

\begin{tabular}{|c|c|c|c|}
\hline \multicolumn{2}{|c|}{ IFDM - Geral (Binned) } & Média & Erro \\
\hline \multirow[t]{3}{*}{ G1 } & Despesa - Saúde & 700,54 & 206,31 \\
\hline & Despesa - Trabalho & 12,43 & 12,67 \\
\hline & Despesa - Educação & 808,94 & 230,87 \\
\hline \multirow[t]{3}{*}{ G2 } & Despesa - Saúde & 675,13 & 212,05 \\
\hline & Despesa - Trabalho & 11,12 & 11,70 \\
\hline & Despesa - Educação & 778,37 & 249,43 \\
\hline \multirow[t]{3}{*}{ G3 } & Despesa - Saúde & 684,03 & 203,06 \\
\hline & Despesa - Trabalho & 10,66 & 11,93 \\
\hline & Despesa - Educação & 760,49 & 239,56 \\
\hline \multirow[t]{3}{*}{ G4 } & Despesa - Saúde & 719,05 & 213,53 \\
\hline & Despesa - Trabalho & 10,04 & 11,33 \\
\hline & Despesa - Educação & 750,83 & 231,44 \\
\hline \multirow[t]{3}{*}{ Total } & Despesa - Saúde & 694,72 & 208,52 \\
\hline & Despesa - Trabalho & 11,06 & 11,90 \\
\hline & Despesa - Educação & 774,70 & 237,89 \\
\hline
\end{tabular}

As médias de cada variável, independentes em cada grupo, não são estatisticamente diferentes. Isso pode ser observado pela Tabela 8 , aonde mostra que as médias e erro colocam os grupos em patamares iguais.

Tabela 8: Estatística descritiva acerca dos grupos.

Fonte: Dados da pesquisa.

\subsection{Resultados da Classificação}

Os resultados para a classificação podem ser observados na 
Research, Society and Development, v. 11, n. 1, e47811125215, 2022

(CC BY 4.0) | ISSN 2525-3409 | DOI: http://dx.doi.org/10.33448/rsd-v11i1.25215

Tabela 9. 
Research, Society and Development, v. 11, n. 1, e47811125215, 2022

(CC BY 4.0) | ISSN 2525-3409 | DOI: http://dx.doi.org/10.33448/rsd-v11i1.25215

Tabela 9: Resultados da classificação dos grupos segundo o modelo elaborado.

\begin{tabular}{|c|c|c|c|c|c|c|c|}
\hline & & IFDM & & ciaçã & po pre & & \\
\hline & & IFDIVI & G1 & $\mathrm{G} 2$ & G3 & G4 & lotal \\
\hline & & G1 & 114 & 205 & 93 & 119 & 531 \\
\hline & & $\mathrm{G} 2$ & 116 & 221 & 80 & 116 & 533 \\
\hline & Contagem & G3 & 86 & 191 & 89 & 166 & 532 \\
\hline & & G4 & 89 & 183 & 74 & 185 & 531 \\
\hline Original & & Casos não agrupados & 0 & 2 & 0 & 1 & 3 \\
\hline Uriginal & & $\mathrm{G} 1$ & 21,5 & 38,6 & 17,5 & 22,4 & 100.0 \\
\hline & & $\mathrm{G} 2$ & 21,8 & 41,5 & 15,0 & 21,8 & 100.0 \\
\hline & $\%$ & G3 & 16,2 & 35,9 & 16,7 & 31,2 & 100.0 \\
\hline & & G4 & 16,8 & 34,5 & 13,9 & 34,8 & 100.0 \\
\hline & & Casos não agrupados & 0 & 66,7 & 0 & 33,3 & 100.0 \\
\hline
\end{tabular}

Fonte: Dados da pesquisa.

Os casos agrupados corretamente podem ser observados, em termos percentuais, nas células grifadas. Pode-se verificar que a taxa de acerto para o modelo classificatório é baixa, com valores inferiores a $42 \%$. A maior taxa de acerto foi para o grupo 2 (41,5\%), seguido pelo grupo 4 (34,8\%), grupo $1(21,5 \%)$ e grupo 3(16,7\%). No agregado de todos os grupos, o acerto foi de $28,6 \%$, ou seja, o modelo apresenta uma precisão muito baixa.

O grau de eficiência do modelo de classificação é atestado pelos resultados da classificação, visíveis na 
Tabela . A eficiência agrupada do modelo foi de 28,6\%, apontando que o modelo apresenta uma precisão bem baixa.

\subsection{Análise Geral do Método Aplicado}

Partindo de uma avaliação global dos dados, desde a elaboração da base de dados representada pela estatística descritiva, já evidenciava-se comportamentos atípicos, pelos valores das despesas em Trabalho serem em sua maioria nulos. Este comportamento influenciou as funções de Fisher que descrevem os grupos categorizados.

Das quatro variáveis selecionadas, somente a variável Despesa - Educação é significante para o modelo, ou seja, somente ela possui impacto na classificação. Entretanto, é necessário novos desenvolvimentos para que seja possível estimar o IFDM com apenas uma das dimensões associadas ao índice.

A eficiência agrupada do modelo foi de $28,6 \%$, apontando que o modelo apresenta uma precisão muito baixa.Pela análise de Lambda de Wilks observou-se que a variável independente educação é a que mais discrimina os grupos, pois o valor $\mathrm{T}$ foi menor que 0,01 .

Por último ficou evidente, que não existe relação de dependência entre as variáveis Despesa-Saúde, DespesaEducação e Despesa-Trabalho (variáveis independentes), com o Índice Firjan de Desenvolvimento Municipal (IFDM), ou seja, os valores investidos nas funções de governo citadas não fazem variar o IFDM, o que vem ao encontro do que os autores Faria et al. (2008), Will (2014) e Dias et al. (2014) apontam em seus estudos a não relação dos gastos em Educação e os índices de eficiência. Os estudos destes autores foram relacionados somente ao gasto com Educação e este estudo denota a não relação também com os gastos com Saúde e Trabalho.

\section{Considerações Finais}

Este estudou avaliou se existe correlação entre os gastos públicos das funções de governo (saúde, educação e trabalho) e o Índice Firjan de Desenvolvimento Municipal (IFDM). Ao analisar os gastos públicos das funções de governo Educação, Saúde e Trabalho dos municípios do Estado de São Paulo, por meio do site das Finanças do Brasil dos Municípios (FINBRA) no período de 2013 a 2016, percebeu-se que a maioria dos municípios de São Paulo não realizam nenhum investimento na função Trabalho.

Os dados apontaram para um maior investimento na Função Educação, seguidos pelos investimentos na Função Saúde. Isto se deve ao que foi imposto por lei na Constituição Federal de 1988, onde foram definidos limites mínimos de gastos com as duas funções, sendo que o percentual mínimo de gasto em Educação (25\% das receitas municipais) é maior do que o mínimo em Saúde (15\% das receitas municipais). A Constituição Federal de 1988 não prevê limites mínimos de gastos com a Função Trabalho, o que pode ter um efeito direto em relação ao não investimento nesta função na maioria dos municípios do estado de São Paulo.

Sabendo que a Função Trabalho está ligada a melhoria das condições de vida da população, sendo considerada como direito social,os resultados deste estudo evidenciam a desatenção dos governos municipais em relação às políticas públicas de emprego e renda, ou mais amplamente, voltados ao desenvolvimento local e à promoção do trabalho, já que os investimentos nesta função são de livre escolha do gestor, deixando a critério de cada gestor municipal a decisão de investir ou não.

De todo modo, em relação à função trabalho é preciso destacar que os indicadores utilizados pelo Índice FIRJAN são oriundos do mercado de trabalho formal, ou seja, não é um indicador de gasto público, como é o caso da função saúde e educação. Para essas duas últimas funções, os municípios têm gastos efetivos e compromissados.

No caso da função Trabalho, além da despesa existência - desde os anos 1980 - de iniciativas e programas municipais de desenvolvimento local, o período recente certamente coloca restrições ainda maiores do ponto de vista do financiamento público de ações de geração de trabalho e renda. 
O IFDM é pautado em pilares distintos para cada uma das suas dimensões (Emprego e Renda, Saúde e Educação) e em cada dimensão, são descritos indicadores para formação do IFDM. Esse indicador pode variar no gradiente que considera estágios de desenvolvimento alto, moderado, regular e baixo. Diante dos resultados obtidos por meio da análise discriminante, o comportamento das variáveis apontou que não existe correlação entre os gastos das funções de governo contemplados neste estudo e o IFDM, ou seja, a variação do IFDM não tem nenhuma dependência com os gastos efetuados nos municípios nas funções aqui estudadas.

Sugere-se para pesquisas futuras que seja realizado uma análise discriminante do IFDM, separando os índices por suas dimensões, relacionando-se com as funções de governo em separado. A maior dificuldade em realizar esta pesquisa foi na construção da base de dados, dada a dimensão da amostra considerada.

\section{Referências}

Arretche, M. T. (1999). Políticas sociais no Brasil: descentralização em um Estado federativo. Revista brasileira de ciências sociais,14, $111-141$.

Avelino, B. C., Bressan, V. G. F., \& da Cunha, J. V. A. (2013). Estudo sobre os fatores contábeis que influenciam o Índice Firjan de Desenvolvimento Municipal (IFDM) nas capitais brasileiras. Revista de Educação e Pesquisa em Contabilidade (REPeC),7(3).

Bellingieri, J. C. (2019). Mensurando o desenvolvimento dos municípios paulistas: uma descrição crítica do IDHM, IFDM e IPRS. Caderno Prudentino de Geografia,1(41), 21-44.

Brasil. Lei no 4.320 (1964). http://www.planalto.gov.br/ccivil_03/leis/14320.htm.

Castro, L. A., Taleires, F. C. D. S. S., \& Silveira, S. S. (2021). Índice de desenvolvimento humano em municípios que possuem sistema integrado de saneamento rural: uma análise comparativa. Ciência \& Saúde Coletiva, 26, 351-357.

Cavalieri, C. H., \& Pazello, E. T. (2005). Efeito distributivo das políticas sociais. Economia do setor público no brasil. Rio de Janeiro: Editora Campus.

Comparato, F. K. (2001). O Ministério Público na defesa dos direitos econômicos, sociais e culturais. Rev. Faculdade Direito Universidade Federal Minas Gerais, 40,67 .

Confins. (2020). O índice FIRJAN de Desenvolvimento Municipal (IFDM). Confins. Revue Franco-Brésilienne de Géographie / Revista Franco-Brasilera de Geografia, 44. https://doi.org/10.4000/confins.26246.

Da Rosa, F. S., Martins, S., Lunkes, R. J., \& Vieira, D. M. (2021). A Influência da Transparência Pública na Relação entre Gestão Fiscal e no Desempenho de Municípios Brasileiros. Desenvolvimento em Questão, 19(54), 262-278.

Da Silva Gomes, E. C. (2019).O direito dos gastos públicos no Brasil. Almedina.

Dantas, M. C., \& Silva, M. V. da. (2019). Analysis of the efficiency of public expenditure with basic education: a study in the Borborema Potiguar-RN microregion. Research, Society and Development, 8(2), e3582782. https://doi.org/10.33448/rsd-v8i2.782.

De Andrade, B. H. S., Costa, A. D. J. B., \& Matos, N. B. (2019). Gasto público e desenvolvimento: uma análise dos municípios brasileiros de grande porte. Revista Uniabeu,12(32).

De Lara Dias, H., Cagnini, W., \& Camargo, S. R. (2014). Análise da eficiência dos gastos públicos com educação nos municípios do Sudoeste do Paraná. InAnais do Congresso Brasileiro de Custos-ABC.

De Oliveira, H. E., \& da Silva, M. D. F. F. (2018). Crescimento versus desenvolvimento local: uma análise entre o pib e a renda média dos municípios do rio grande do norte. Empírica BR-Revista Brasileira de Gestão, Negócio e Tecnologia da Informação, 1(1), 268-295.

De Sousa, A. M., Da Rosa, F. S., \& Ribeiro, A. M. (2020). Influência dos gastos públicos no crescimento e desenvolvimento econômico: uma análise em municípios de Santa Catarina. GCG: revista de globalización, competitividad y gobernabilidad,14(1), 62-77.

De Sousa, M. G., dos Santos, C. M. V., Alves, A. T., \& do Carmo Filho, M. M. (2021). Uma análise da eficiência dos gastos públicos com educação nos municípios do Estado do Amazonas no período de 2013 a 2017. Revista Ambiente Contábil-Universidade Federal do Rio Grande do Norte-ISSN 2176-9036, $13(1), 222-243$

Diel, E. H., Diel, F. J., Schulz, S. J., Chiarello, T. C., \& da Rosa, F. S. (2014). Desempenho de municípios brasileiros em relação à estratégia de investimento público em educação. Desenvolvimento em Questão, 12(26), 79-107.

Diel, E. H., Diel, F. J., Schulz, S. J., Chiarello, T. C., \& da Rosa, F. S. (2014). Desempenho de municípios brasileiros em relação à estratégia de investimento público em educação. Desenvolvimento em Questão,12(26), 79-107.

Do Brasil, Senado Federal. (1998) Constituição da República Federativa do Brasil. Brasília: Senado Federal, Centro Gráfico.

Duarte, V. N., \& Alves, L. R. (2019). Índice Firjan de desenvolvimento municipal no estado de mato grosso do sul: o que mostram os números?.X Seminário Internacional sobre Desenvolvimento Regional. 
Faria, F. P., Jannuzzi, P. D. M., \& Silva, S. J. D. (2008). Eficiência dos gastos municipais em saúde e educação: uma investigação através da análise envoltória no estado do Rio de Janeiro.Revista de administração pública,42, 155-177.

Ferreiro Lapatza, J. J. (2004). Curso de Derecho financiero español “, 24 edición.Revisada y puesta al día, Marcial Pons.

FIRJAN. Índice FIRJAN de Desenvolvimento Municipal (IFDM). Disponível em: <http://www.firjan.com.br/ifdm/>.

Forte, MO e Vieira, LME da R. (2021). A eficiência no planejamento orçamentário influencia a situação financeira dos estados brasileiros?. Research, Society and Development, 10 (11), e455101119947. https://doi.org/10.33448/rsd-v10i11.19947

Giacomoni, J. (2003). Orçamento público. Atlas.

Goulart, J. L., Troian, A., \& Quispe, J. N. (2020). Observatórios Sociais e sua Importância para a Gestão Pública na Região Sul do Brasil. Desenvolvimento em Questão,18(51), 113-128.

Höfling, E. D. (2001). Estado e políticas (públicas) sociais. Cadernos Cedes,21, 30-41.

IFDM | Índice FIRJAN de Desenvolvimento Municipal: Consulta. (n.d.). www.firjan.com.br. http://www.firjan.com.br/ifdm/.

Ipea. (2012). Políticas sociais: acompanhamento e análise.

Jesus, A. C. S. de, Dantas, A. L. F., \& Silva, M. V. da. (2019). The efficiency of public spending in education: an evaluation of the Serideo Potiguar region (2008-2015). Research, Society and Development, 8(12), e138121602. https://doi.org/10.33448/rsd-v8i12.1602

Lepchak, A.., Lima Filho, SS., Silva, E. O. da., \& Peixe, BCS. (2021). Análise da eficiência no uso dos recursos de saúde nos maiores municípios brasileiros. Research, Society and Development, 10 (15), e382101522669. https://doi.org/10.33448/rsd-v10i15.22669

Magalhães, J. C. (2007). Emancipação político-administrativa de municípios no Brasil. Dinâmica dos municípios. Brasília: IPEA, $15-52$.

Massaú, G., \& Rafagnin, T. R. (2021). Análise dos conceitos de direitos sociais oriundos da doutrina brasileira. Sociedade em Debate, $27(1), 250-262$.

Matias-Pereira, J. (2012). Manual de gestão pública contemporânea: inclui análise dos efeitos das mudanças de paradigmas na administração pública brasileira. In Manual de gestão pública contemporânea: inclui análise dos efeitos das mudanças de paradigmas na administração pública brasileira(pp. xvi$310)$.

Mello, G. R., Slomski, V., \& Corrar, L. J. (2005). Estudo dos reflexos da lei de responsabilidade fiscal no endividamento dos estados brasileiros. Journal of Accounting, Management and Governance, 8(1).

Monteiro Neto, A., Costa, M. A., Resende, G. M., Mendes, C. C., \& Galindo, E. P. (2017). Desenvolvimento territorial no Brasil: reflexões sobre políticas e instrumentos no período recente e propostas de aperfeiçoamento.

Oliveira, L. S. D. D. (2016). As despesas públicas municipais como determinante no desenvolvimento humano.

Pinheiro, A. C., Barbosa, W. L. R., \& Sobrinho, M. V. (2020). Gasto de Recurso Público e seu Impacto no Desenvolvimento Territorial: o caso de Brumadinho. Research, Society and Development, 9 (2), 44.

Procopiuck, M., Machado, E. T., Rezende, D. A., \& Bessa, F. L. B. N. (2007). O Plano Plurianual Municipal no sistema de planejamento e orçamento brasileiro. Revista do Serviço Público, 58(4), 397-415.

Rezende, A. J., Slomski, V., \& Corrar, L. J. (2005). A gestão pública municipal e a eficiência dos gastos públicos: uma investigação empírica entre as políticas públicas e o índice de desenvolvimento humano (IDH) dos municípios do Estado de São Paulo. Revista Universo Contábil, 1 (1), $24-40$.

Rezende, F. (2006). Finanças públicas. São Paulo: Atlas.

Rezende, F. D. C. (1997). Descentralização, gastos públicos e preferências alocativas dos governos locais no Brasil:(1980-1994). Dados,40, 264-279.

Rezende, F., Cunha, A., \& Cardoso, R. L. (2010). Custos no setor público. Revista de Administração Publica-RAP, 44 (4), $789-791$.

Riani, F. (2000). Economia Do Setor Público: Uma Abordagem Introdutória. Grupo Gen-LTC.

Sales, S. H. A. (2019). O desenvolvimento socioeconômico em municípios receptores de parques eólicos: uma análise com índices firjan. RDE-Revista de Desenvolvimento Econômico, 2(43).

Santos Filho, M. R. D. (2010). Desenvolvimento humano dos municípios baianos: uma avaliação a partir de indicadores sociais e das demonstrações contábeis.

Saraiva, J. D. S. (2019). Crescimento e desenvolvimento econômico dos municípios do estado do Pará no período 2005 -2016.

Scarpin, J. E., \& Slomski, V. (2007). Estudo dos fatores condicionantes do índice de desenvolvimento humano nos municípios do estado do Paraná: instrumento de controladoria para a tomada de decisões na gestão governamental. Revista de administração pública,41, 909-933.

Silva, M. C. A, V. A. D. (2018). Desenvolvimento municipal: efetividade ou inércia nos municípios do Estado do Rio Grande do Norte (2005-2013). Revista Contabilidade, Ciência da Gestão e Finanças. 6 (2). 
Research, Society and Development, v. 11, n. 1, e47811125215, 2022

(CC BY 4.0) | ISSN 2525-3409 | DOI: http://dx.doi.org/10.33448/rsd-v11i1.25215

Silva, R. F. G., \& de Brito, L. A. P. F (2019). Crescimento econômico e desenvolvimento econômico: uma análise pelo índice FIRJAN e PIB per capita do município de São José dos Campos-SP.Gestão e Desenvolvimento em Revista, 5(2), 59-68.

Silva, V. B. D., Oliveira, E. A. D. A. Q., \& Carniello, M. F. (2021). Índice Firjan de desenvolvimento e indicações geográficas de municípios da região nordeste do brasil: uma análise do desenvolvimento regional. X Seminário Internacional sobre Desenvolvimento Regional.

Slomski, V., \& Scarpin J. E. (2006). Estudo dos fatores condicionantes do Índice de Desenvolvimento Humano nos municípios do Estado do Paraná: instrumento de controladoria para a tomada de decisões na gestão governamental. In:Congresso USP Controladoria e Contabilidade.

Soares, D. J. M., Soares, T. E. A., Santos, M. C. de S., \& Santos, W. dos. (2020). Analysis of the efficiency of public expenditure on education in capixaba municipalities. Research, Society and Development, 9(7), e756974906. https://doi.org/10.33448/rsd-v9i7.4906.

Soares, J. R., Raupp, F. M., \& Tezza, R. (2021). Qualidade do gasto público nos municípios de santa catarina. Contabilidade Vista \& Revista, 32 (3), $165-194$.

Sun, S., \& Andrews, R. (2020). The determinants of fiscal transparency in Chinese city-level governments. Local Government Studies,46 (1), $44-67$.

Torres, R. L. (1996). Reforma Fiscal do Estado ou Reforma do Estado Fiscal. Rio de Janeiro.

Whiteley, P. F. (2000). Economic growthand social capital. Political studies, 48 (3), 443-466.

Will, A. R. (2014). Eficiência dos estados brasileiros nos gastos com educação: um estudo comparativo de recursos utilizados e resultados alcançados.

Zoghbi, A. C. P., Matos, E. H. C. D., Rocha, F. F., \& Arvate, P. R. (2009). Mensurando o desempenho e a eficiência dos gastos estaduais em educação fundamental e média. Estudos Econômicos (São Paulo), 39, 785-809. 